\title{
Human Trust in Robots When Performing a Service
}

\author{
Kurt Byrne \\ Alliance Manchester Business School \\ The University of Manchester \\ Manchester, UK
}

\author{
César A. Marín \\ Information Catalyst for Enterprise Ltd \\ Crewe, UK \\ cesar.marin@informationcatalyst.com
}

\begin{abstract}
The presence of robots is becoming more apparent as technology progresses and the market focus transitions from smart phones to robotic personal assistants such as those provided by Amazon and Google. The integration of robots in our societies is an inevitable tendency in which robots in many forms and with many functionalities will provide services to humans. This calls for an understanding of how humans are affected by both the presence of and the reliance on robots to perform services for them. In this paper we explore the effects that robots have on humans when a service is performed on request. We expose three groups of human participants to three levels of service completion performed by robots. We record and analyse human perceptions such as propensity to trust, competency, responsiveness, sociability, and team work ability. Our results demonstrate that humans tend to trust robots and are more willing to interact with them when they autonomously recover from failure by requesting help from other robots to fulfil their service. This supports the view that autonomy and team working capabilities must be brought into robots in an effort to strengthen trust in robots performing a service.
\end{abstract}

\section{Introduction}

In the 1960's industrial robotics started becoming commercially available to big industries, including car manufacturers, to mass produce with an accuracy of $1 /$ 10,000th of an inch [1]. There has been a revolutionary expansion in robotics that only recently they started being ingrained in people's lives in the form of service providers from rehabilitation [2] to entertainment [3].

A service robot is a robot that helps or does work for someone that is useful to the well-being of people and equipment in a semi or fully autonomous way, excluding manufacturing operations [4]. By doing this, robots are providing a service as humans can instead engage in thoughtful experiences as opposed to monotonous tasks ranging from serving drinks to mining rock [5]. There are four societal paradigm shifts because the context in which robots operate have changed over the years [1]:

Paradigm shift 1) As humans we realise that we could nullify the need to be involved with dirty, dull, dumb or dangerous tasks. Instead we make robots replace us doing so. Paradigm shift 2) As consumers we want high quality customisable products at a low cost. We use robots in an industrial context to mass produce products in a flexible and reconfigurable manner. Paradigm shift 3)

This work was partially funded by the European Commission through the project DIGICOR (http://digicor-project.eu/) under grant agreement No. 723336
Robots are entwined in our daily lives. This requires for the most natural and simple interactive methods to be developed in order to facilitate human-robot interactions (HRI). Paradigm shift 4) A complete rethinking of how health care is conducted is necessary, with the goal of producing a fully competent robotic companion to fully support our ageing population.

The third paradigm shift is the one our society is living today and it still requires a wealth of research to help humans interact with robots on a subconscious level, much like the way we do with other humans. Research to fulfil this from the robots' side is ongoing, e.g. [6]-[8]. However there is little input from the human side. This calls for further studies on HRI and in particular studies regarding human trust in robots when providing a service.

This paper presents a study on HRI paying particular attention to how trust is affected in humans when a robot achieves different levels of completion of a requested service. Three groups of human participants were exposed to robots performing a requested service. Data collected during these expositions were analysed to gain an insight on how trust was affected after the exposure to the robots. Our findings support the view that autonomy and robotto-robot team work capabilities must be embedded into robots in order to increase trust and acceptance of robots when performing a service for humans.

The rest of the paper is structured as follows: Section II presents the background and related work in the area. Section III describes the framework used for this research. Section IV presents the experiment carried out with human participants and robots. Section V discusses the implications and limitations of the findings. Finally, section VI concludes the paper with further research.

\section{Background}

When industrial robots and computers first appeared as innovations, they were inaccessible because they required trained individuals to operate them. In contrast, service robots are required to be controlled by unskilled individuals to complete specific human requests [2].

Japan have been more accepting of service robots in their everyday life as opposed to any other location in the world [9]. Japan employees are guaranteed a "lifetime employment system" which enables them to be repositioned within their organisation if their current role is later 
fulfilled by a robot. Therefore, robot introduction does not bring along negative stigmas but instead new, exciting opportunities to allow individuals to develop themselves because of the assignment of a new role [9].

In the European Union the introduction of robots in the workplace is viewed as a threat of losing people's jobs. Sweden rejected a proposal to guarantee its citizens a basic income no matter their status of employment regarding workplace automation ${ }^{1}$. $79 \%$ of voters rejected an unconditional payment of $\$ 2,560$ a month which could have provided relief to any individuals whose role had been made defunct by robots. This rejection suggests that western cultures have different attitudes to robotics as opposed to Japan, but it does raise an important issue in that a fifth of Swedish inhabitants are concerned about the ever-increasing autonomy of the workplace through robotic application.

Nevertheless, the normalisation of service robots is becoming ever more apparent in recent years. Robots have become $75 \%$ cheaper in the past few years; it is understandable to as why service robots will soon become extremely accessible [10]. This raises a range of ethical, technical and societal issues that need to be addressed.

\section{A. Human-Robot Interactions (HRI)}

HRI has long been neglected by the scientific community up until the year of 2006 when the literature began to rapidly expand, cf. [11]-[13]. The area has only newly emerged in the past decade due to the parallels found with the discipline of human-computer interaction (HCI). Therefore, the need to research specifically humans interacting with robots is huge and relatively untapped. HRI is a combination of inter-disciplinary areas including computer science, engineering, robotics, and psychology; investigating social behaviours and intelligence in both natural and artificial systems. However, it is the interaction with people which is the defining area of HRI, which can be categorised into three distinct directions [11]:

- Robot cognition-centred HRI. This direction focuses on the robot as an intelligent system, where it makes its own decisions to tackle problems in a context.

- Robot centred HRI. This direction regards the skills a robot must have in order to survive in its environment; the robot is viewed as a creature relying on its own autonomous actions to proceed with a task.

- Human centred HRI. The final direction is concerned with how a robot can fulfil its human counterpart's requests in a way that is accepted in a socially comfortable manner. This is where this paper fits.

\section{B. Behaviour and Trust}

It had once been assumed that it was both the appearance and behaviour of a robot what influenced interactions between humans and robots, until [14] found out that

\footnotetext{
${ }^{1}$ http://in.reuters.com/article/swiss-vote-idINKCNOYROIJ Reuters India. Last accessed 10/Jan/2018.
}

appearance distorts our interpretation of robot behaviour as participants fixated more on eyes and nose than in any other part of the robot. There has also been evidence from the psychological community's observations of infants in that they follow the gaze of a novel object that was decorated with facial features [15]. Further work has also documented that a 1-millimetre repositioning of a facial feature will cause a human to view a formerly attractive face as unattractive [16]. It has been demonstrated that children and adults alike are able to transfer human aesthetics over to unnatural objects [17]. It is safe to assume that a robot designed with human like features could illicit a positive reception from a human.

To further facilitate human-robot relations in the future it is necessary to develop robots that interact socially with humans and produce empathetic behaviours using e.g. vocal feedback and facial expressions [18]. A pilot study compared an empathetic robot to a more neutrally responsive robot and the results suggest that participants working with the more empathetic robot were more likely to perceive the robot as a friend [18]. This perception of relationship development supports the view that a robot must mirror a human's vulnerability and become human empathetic to truly transform robots from a tool into a companion [19].

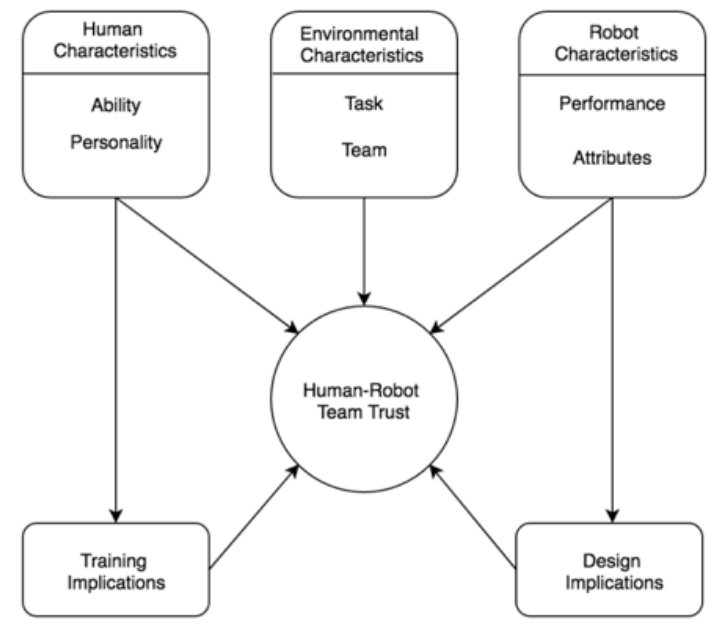

Fig. 1. Triadic model for Human-Robot team trust [20].

As the capabilities of service robots continue to grow, so does their possibility of becoming fully fledged team members. The field of robotics has already decided that robots can participate in human teams, with a range of studies documenting examples of human-robot trust in critical situations such as warfare and rescue operations, e.g. [12], [21], [22]. The most important characteristics to develop strong levels of trust in HRI within the context of humanrobot teams are identified by [12] where a meta-analysis allowed for the development of a perception framework, later refined by [20]. The refined framework (see Fig. 1) 
presents different characteristics of humans, robots and the surrounding environment as the basis for trust in human-robot teams. Specifically, robots' performance and reliability are found to be the most important factors in developing trust in critical situations where humans and robots are part of the same team [12], [20].

Yet not every HRI is expected to be in the context of teams. Service robots can also be seen as providers of one-off services to humans. In the context of service provision, e.g. delivering an item or fetching materials, unexpected circumstances external to the robot might appear rendering the service unfulfilled. This will surely have an impact on how humans will trust robots in the future. Additionally, a robot that exceeds human expectations on service fulfilment will also have an impact on that trust. It is of great importance to attain this understanding in order to close the gap in HRI when robots provide one-off services for humans.

\section{Perception Framework of Trust for Service Robots}

Inspired by [20] and motivated by the need of a framework of trust for robots in the context of service provision, we present a perception framework of trust for service robots. Our framework focuses on both human and robot attributes intrinsic with the potential service fulfilment level rather than those needed for human-robot teams, cf. [12], [20], [23]. Figure 2 presents a graphical representation of our framework. The novelty of our approach is its application to service provision where less emphasis is made on training implications and the human need to feel that robots look after them as in the Triadic model [12], [20]; additionally, sociability has been added as suggested by [23]. Key attributes are classified according to attributes and characteristics presented by [12], although they were not defined. Here we define these attributes as follows:

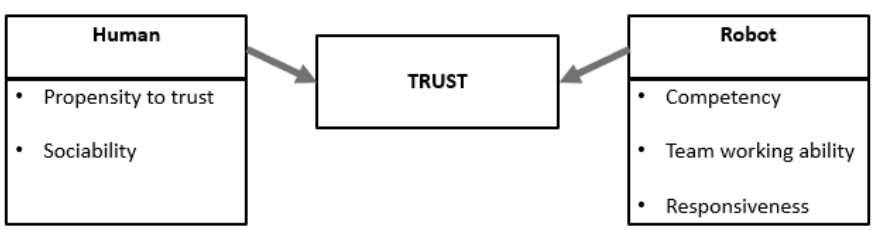

Fig. 2. Perception framework of human trust in service robots.

1) Propensity to Trust is a human characteristic that refers to the tendency of a human to believe a robot can fulfil a requested service. 2) Sociability is another human characteristic (as suggested by [23]) that refers to the tendency of a human to assign social traits to a robot, thus giving the impression the robot can interact with humans. 3) Competency is a robot characteristic that refers to the perceived robot capacity to successfully carry out a given service request. 4) Team Working Ability is a robot characteristic that relates to the perceived capacity of a robot to work in a team with other robots, as opposed to human-robot teams, cf. [12]. Finally, 5) Responsiveness is another robot characteristic that consists of the robot's ability to react promptly when a service is requested.

To instantiate and evaluate our approach, an experiment was developed to expose humans to different levels of service completion performed by a robot. We measure each of the attributes of our approach with a questionnaire given to human participants before and after being exposed to robots. The most common task seen in the literature is a search-and-retrieve task using one or more robots, cf. [4], [12], [24]. Despite the perceived simplicity of the task, the experiment allowed the opportunity for humans with no experience with robots and with no technical background to be exposed to robots performing a requested service. Two LEGO Mindstorm robots ${ }^{2}$ were used for the experiment. It was decided to use these small robots because according to [25] 1) humans are more likely to work with smaller robots since they seem safer; and 2) the idea of robots coordinating themselves with as little input as possible is appealing.

Figure 3 presents the robots used. The first robot, named "Gripper", has a function to grab objects with its large front facing claw, powered by the medium motor capable of $250 \mathrm{rpm}$. The second robot, named "Locator", is fitted with a colour sensor and an infrared sensor. The colour sensor could detect up to 7 different colours, whilst the infrared sensor could detect a homing beacon within proximity of $70 \mathrm{~cm}$. The physicality of the infrared sensor was comparable to a human face, and was intentionally fitted to the front of the robot to elicit empathetic responses from participants.

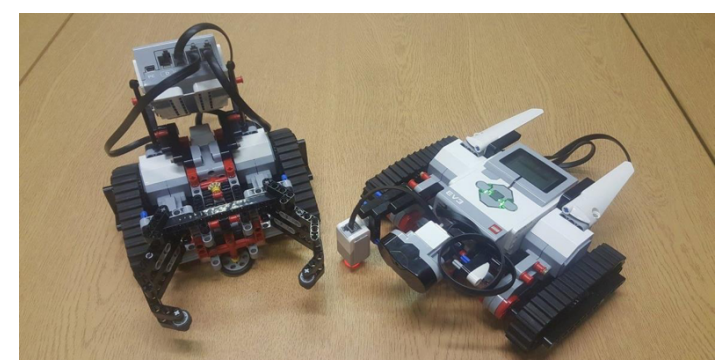

Fig. 3. Gipper (left) and Locator (right).

\section{Experiment on Human Trust in Service Robots}

The purpose of the experiment is to test the impact on each attribute of our framework when human participants are exposed to different levels of service completion. The experiment then consists of exposing three groups of human participants to one scenario each where a robot has to provide a service with different levels of service completion namely a) failure, b) pass, c) team success. At the start of all scenarios the Gripper is located at one end of a table and the Locator is situated between the middle

\footnotetext{
${ }^{2}$ https://www.lego.com/en-gb/mindstorms /
} 
and the opposite end of the same table. The experiment then consists of having the participant "ask" the Gripper, via its small interface, to collect a cup from the middle of a table and delivering it to the participant, see Fig. 4. The scenarios are described as follow:

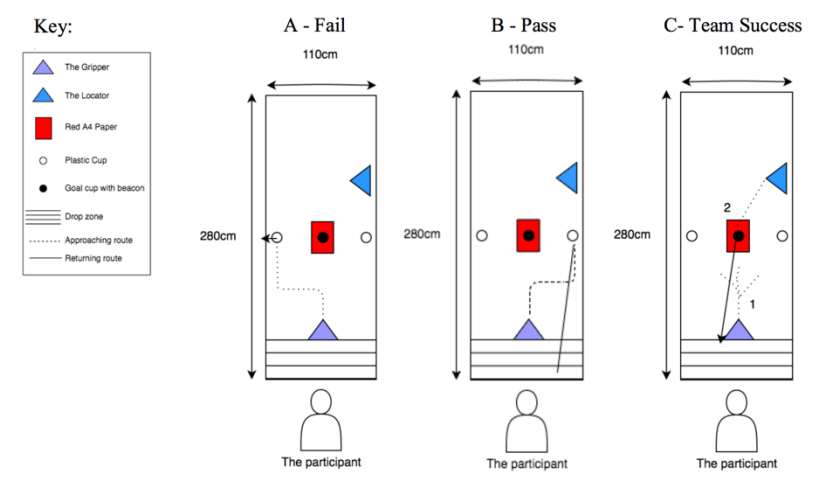

Fig. 4. Experiment with three scenarios: a) failure, b) pass, c) team success.

a) Failure scenario: The Gripper robot manoeuvres its way to the cup on the left, picks it up and rotates 90 degrees counter clockwise, followed by dropping the cup off the table. It then vocally apologises by saying "sorry". The Gripper robot would then remain motionless signalling the end of the scenario.

b) Pass scenario: The Gripper robot travels to the cup on the right and grasps the cup; it then rotates 180 degrees counter clockwise and travel back to the drop zone where the participant is sitting. The robot successfully delivers the cup to the participant and robot vocalises a celebration signalling the end of the scenario.

c) Team success scenario: The Gripper robot approaches the cup in the middle but it banks 45 degrees either way, vocalising "uh-oh", thus giving the appearance of being confused. The Gripper robot then communicates via Bluetooth with the Locator robot asking for help. The Locator navigates towards the middle cup by following the beacon sitting inside the cup. Using its camera to detect the red paper under the cup in order to detect when to stop, the Locator informs the Gripper of its finding who immediately proceeds to pick up the cup then returns to deliver it to the participant. This gives the appearance to the participant that the Gripper robots asked for help when needed and both robots managed to work together as a team to fulfil a service.

A total of 45 participants, split in three different groups, were involved in this study consisting of 32 males and 13 females, all ranging from ages 18 to 30 years old from a range of academic backgrounds. The gender imbalance of the participants is related to robotics being a field previously identified with a discerning gender gap ${ }^{3}$. Furthermore, the age limit for participation was strictly

\footnotetext{
${ }^{3}$ http://www.pbs.org/newshour/making-sense/truth-womenstem-careers/ Why the STEM gender gap is overblown.
}

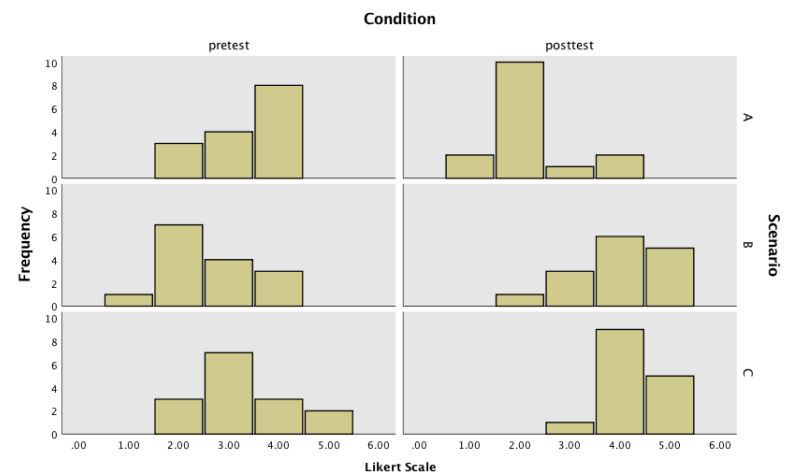

Fig. 5. Propensity to Trust likert scores across scenarios.

limited to 30 years old due to older generations having different predispositions to technology and would likely produce seemingly anomalous results [26].

To evaluate our approach, we first presented a questionnaire to each participant where, using a Likert scale, we collected their views on robots regarding (see Fig. 2) 1) propensity to trust, 2) sociability, 3) competency, 4) team working ability, and 5) responsiveness. We then proceeded with the task where participants had to "ask" the robot, by selecting an option on an interface on the Gripper, to get a plastic cup. The Gripper robot would proceed according to the scenario. Afterwards a second questionnaire was presented to the participant with exactly the same questions as before. This enables us to capture any changes in their perception towards the robot due to recent experience in a specific scenario.

\section{A. Results on Propensity to Trust}

Participants were asked to indicate their level of agreement/disagreement to the sentence "I trust the robots to conduct a service" representing the human attribute Propensity to Trust. Figure 5 compares the Likert scores of the three scenarios. The failure scenario (a) is the only one where answers in the second questionnaire shifted towards a disagreement to the sentence due to the failure itself. For pass scenario (b) and team success (c) there was a shift towards a total agreement to the sentence due to experiencing a success. Notice that the shift in scenario team success (c) is sharper.

\section{B. Results on Sociability}

Participants were asked to choose their level of agreement/disagreement to the sentence "The robots are sociable". Figure 6 presents a comparison of participant's answers in Likert scores of the three scenarios. The failure scenario (a) has its responses spread across the Likert scale in the second questionnaire. This result is the reflection of the vocalisation that robots are capable of because participants perceived that as a social skill. For the other two scenarios the shift towards a total agreement to the 


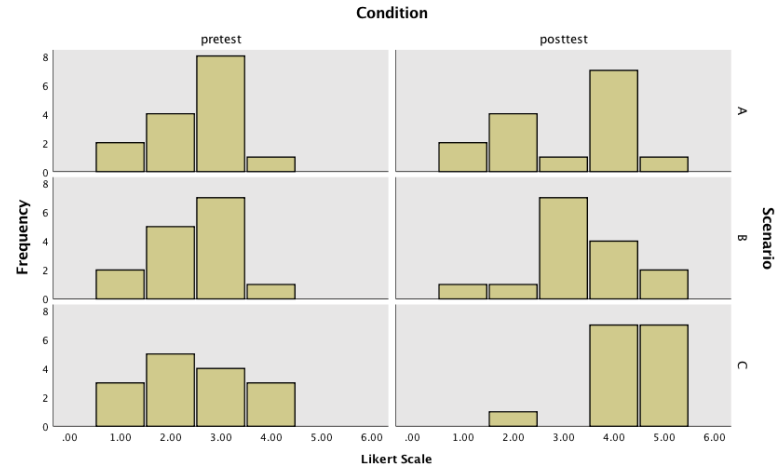

Fig. 6. Comparison of sociability scores across scenarios.

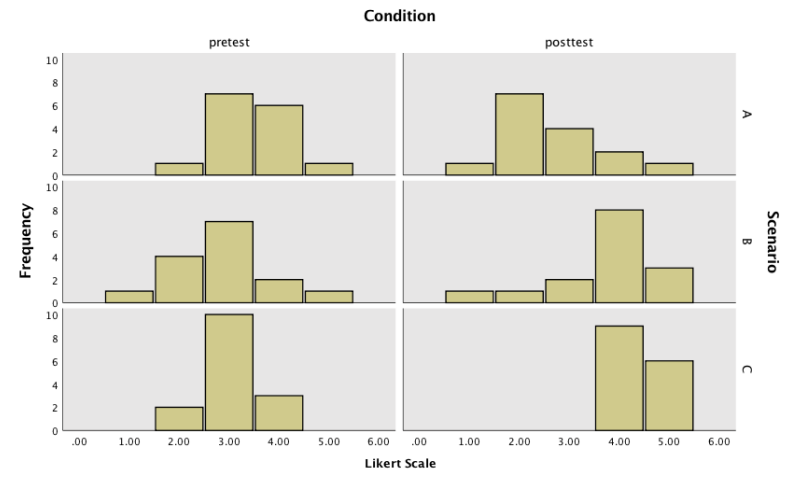

Fig. 7. Comparison of competency scores across scenarios.

sentence is noticeable due to the actual success. Still notice the sharper shift for scenario (c).

\section{Results on Competency}

For Competency, participants were asked to indicate their level of agreement/disagreement to the sentence "The robots are competent". Figure 7 depicts the results in Likert scores of the three scenarios. The failure scenario a) has a clear shift towards total disagreement in the Likert scale as expected because the robot failed the task. Both scenarios pass (b) and team success (c) have a shift towards total agreement. In particular for team success scenario (c), this shift is sharper and focused on scores 4 and 5 only. This is due to the view that team work is regarded as highly competent.

\section{Results on Team Working Ability}

For this attribute, participants were asked to choose their level of agreement/disagreement to the sentence "Robots perform better in a team". Figure 8 presents the results of the Likert scores of the three scenarios. In this case, both the failure scenario (a) and the pass scenario (b) show no significant change since participants did not receive any stimulus for changing their perception, i.e. they only saw the second robot but did not see it in collaboration with the first one. As expected, team success

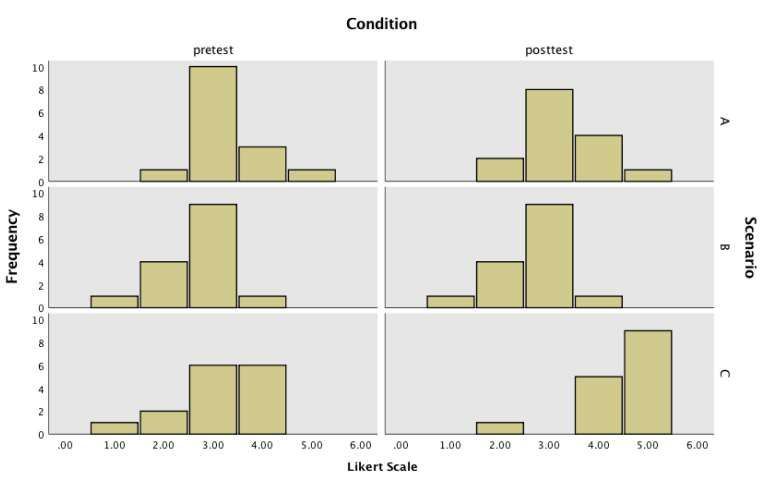

Fig. 8. Comparison of team work ability scores across scenarios.

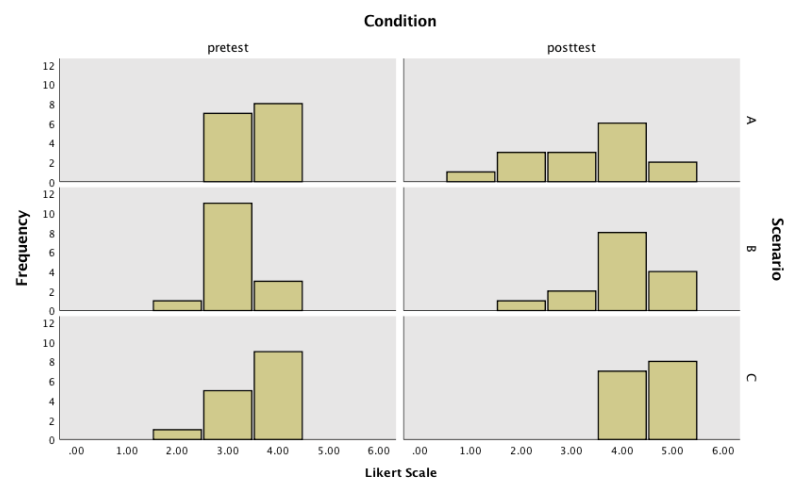

Fig. 9. Comparison of responsiveness scores across scenarios.

scenario (c) shows a sharp shift towards total agreement. This supports the idea that autonomous teams of robots increase human confidence towards them.

\section{E. Results on Responsiveness}

Participants were asked to indicate their level of agreement/disagreement to the sentence "Robots are responsive" for this particular attribute. Figure 9 depicts the results of the Likert scores across the three scenarios. Failure scenario (a) shows a spreading of responses in all directions but in average responses remain with 3 points in the Likert scale. This is due to a combination of perceptions between a quick reaction of the robot at the moment the service is requested and the failure itself. Pass scenario (b) is slightly increased. Team success scenario (c), see Fig. 9, has its scores concentrated in Agree and Total Agree.

\section{Discussion}

Whilst there has been some research on human-robot teams and the factor of trust, cf. [12], [23], there has not been any significant studies that have demonstrated the distinct effects of different levels of service completion on a human's perception when interacting with service robots. The inclusion of three distinct scenarios in our work is used to indicate what attributes of human trust in service 
robots are more likely to change in reaction to different levels of service completion. This supports our claim on the novelty of our approach and the emphasis that should be put on robots as service providers.

Team success scenario (c) produced consistently higher responses from the participants after being exposed to two robots working as a team. They were more likely to apply human aesthetics to the robots operating in this condition; $54 \%$ of participants in this condition referred to the "face" of the Locator being a major contributory factor to their interaction. Furthermore, considering that the robots vocalised actions and situations, even if very small, $80 \%$ of participants mentioned that this feature exceeded their expectations and gave them confidence in their service ability. These were two distinct features that were unavailable in the other scenarios and this emphasises the importance that human empathetic features have in the development of service robots of the future. The results in this study provide further support in HRI to the works conducted by [18], [19], which also stress the importance of empathetic aesthetics in robotic development to facilitate an interactive response from humans.

A limitation of this study is the inability to directly compare the results acquired from our service robots with other studies that have tested different forms of service robot. The conceptual idea behind service robots is that they are personal servants and therefore are developed in many shapes and sizes with a variety of behaviours. The robots in this study cannot be accurately compared to humanoid robots such as the WABOT II [3] to draw any valid similarities or differences, because their purposes do not collate in any way.

\section{Conclusion}

In this paper we present a study on human trust in robots in the context of service provision. We introduce the perception framework of human trust in service robots inspired by [12], [23]. The novelty of our approach is the contextualisation of trust when humans request oneoff services from robots. As identified by our results, service completion is important but teams of robots helping each other fulfil a requested service influences more the level of trust a human has on robots. This feature must be explored more in future research. Additionally, a comparison of this approach with human-to-human service provision must be considered, and results channeled to enhance the perception framework of trust for service robots.

\section{References}

[1] H. Van Brussel, "Robots: The sorcerer's apprentice broom?" European Review, vol. 20, pp. 356-364, 2012.

[2] G. Bolmsjo, H. Neveryd, and H. Eftring, "Robotics in rehabilitation," IEEE Transactions on Rehabilitation Engineering, vol. 3, pp. 77-83, 1995.

[3] T. N. Hornyak, Loving The Machine, 1st ed. Tokyo: Kodansha International, 2006.
[4] G. Bekey, Autonomous robots: from biological inspiration to implementation and control. MIT press, 2005.

[5] D. Hainsworth, "Teleoperation user interfaces for mining robotics," Autonomous Robots, vol. 11, pp. 19-28, 2001.

[6] M. M. Scheunemann, K. Dautenhahn, M. Salem, and B. Robins, "Utilizing bluetooth low energy to recognize proximity, touch and humans," in 25th IEEE International Symposium on Robot and Human Interactive Communication (RO-MAN). IEEE, 2016, pp. 362-367.

[7] L. Pessoa, "Do intelligent robots need emotion?" Trends in cognitive sciences, vol. 21 , no. 11 , pp. 817-819, 2017.

[8] B. Leme, C. Piezzo, M. Hirokawa, and K. Suzuki, "Design of a cloud-based robotic platform for accompanying and interacting with humans," in International Conference on Social Robotics. Springer, 2017, pp. 262-271.

[9] F. L. Schodt, Inside The Robot Kingdom, 1st ed. Tokyo: Kodansha International, 1990.

[10] J. Cosgrave, "Got $\$ 93,000$ to spare? robots get cheaper," CNBC. Available at: http://www.cnbc.com/2014/05/01/got-93000-tospare-robots-getting-cheaper.html, 2014.

[11] K. Dautenhahn, "Socially intelligent robots: dimensions of human-robot interaction," Philosophical Transactions of the Royal Society B: Biological Sciences, vol. 362, pp. 679-704, 2007.

[12] P. Hancock, D. Billings, K. Schaefer, J. Chen, E. De Visser, and R. Parasuraman, "A meta-analysis of factors affecting trust in human-robot interaction," Human Factors: The Journal of the Human Factors and Ergonomics Society, vol. 53, pp. 517-527, 2011.

[13] T. Sheridan, "Human-robot interaction status and challenges," Human Factors: The Journal of the Human Factors and Ergonomics Society, vol. 58, pp. 525-532, 2016.

[14] T. Minato, M. Shimada, H. Ishiguro, and S. Itakura, "Development of an android robot for studying human-robot interaction," Innovations in applied artificial intelligence, pp. 424-434, 2004.

[15] S. Johnson, V. Slaughter, K. Collins, J. Tyan, and S. Carey, "Whose gaze will infants follow? features that elicit gazefollowing in twelve-month-olds," Infant Behavior and Development, vol. 19, pp. 527-, 1996.

[16] N. L. Etcoff, Survival Of The Prettiest, 1st ed. New York: Anchor Books, 2000

[17] T. Fong, I. Nourbakhsh, and K. Dautenhahn, "A survey of socially interactive robots," Robotics and autonomous systems, vol. 42, pp. 143-166, 2003.

[18] I. Leite, A. Pereira, S. Mascarenhas, C. Martinho, R. Prada, and A. Paiva, "The influence of empathy in human-robot relations," International journal of human-computer studies, vol. 71, pp. 250-260, 2013.

[19] M. Coeckelbergh, "Artificial companions: Empathy and vulnerability mirroring in human-robot relations," Studies in ethics, law, and technology, vol. 4, pp. 2-, 2011.

[20] D. R. Billings, K. E. Schaefer, J. Y. Chen, V. Kocsis, M. Barrera, J. Cook, M. Ferrer, and P. A. Hancock, "Human-animal trust as an analog for human-robot trust: A review of current evidence," University of Central Florida Orlando, Tech. Rep., 2012.

[21] C. Breazeal and G. Hoffman, "Collaboration in human-robot teams," in AIAA 1st Intelligent Systems Technical Conference, sep 2004, pp. 6434-.

[22] V. Groom and C. Nass, "Can robots be teammates?: Benchmarks in human-robot teams," Interaction Studies, vol. 8, pp. 483-500, 2007.

[23] K. Schaefer, "The perception and measurement of humanrobot trust," Ph.D. dissertation, University of Central Florida, Orlando, Florida. USA, 2013.

[24] Y. Pyo, K. Nakashima, S. Kuwahata, R. Kurazume, T. Tsuji, K. Morooka, and T. Hasegawa, "Service robot system with an informationally structured environment," Robotics and Autonomous Systems, vol. 74, pp. 148-165, 2015.

[25] R. Wood and C. Walsh, "Smaller, softer, safer, smarter robots," Science Translational Medicine, vol. 5, 2013.

[26] N. Chappell and Z. Zimmer, "Receptivity to new technology among older adults," Disability and rehabilitation, vol. 21, pp. 222-230, 1999. 